\title{
Low-temperature transport in highly boron-doped nanocrystalline diamond
}

\author{
P. Achatz, ${ }^{1,2,3, *}$ W. Gajewski, ${ }^{1}$ E. Bustarret, ${ }^{2}$ C. Marcenat,${ }^{3}$ R. Piquerel, ${ }^{3}$ C. Chapelier, ${ }^{3}$ T. Dubouchet, ${ }^{3}$ O. A. Williams,,${ }^{4,5}$ \\ K. Haenen, ${ }^{4,5}$ J. A. Garrido, ${ }^{1}$ and M. Stutzmann ${ }^{1}$ \\ ${ }^{1}$ Walter Schottky Institut, Technische Universität München, Am Coulombwall, D-85748 Garching, Germany \\ ${ }^{2}$ Institut Néel, CNRS et Université Joseph Fourier, B.P. 166, 38042 Grenoble Cedex 9, France \\ ${ }^{3}$ CEA, INAC, SPSMS, LaTEQS, F-38054 Grenoble Cedex 9, France \\ ${ }^{4}$ Institute for Materials Research, Universiteit Hasselt, Wetenschapspark 1, 3590 Diepenbeek, Belgium \\ ${ }^{5}$ Division IMOMEC, IMEC vzw, Wetenschapspark 1, 3590 Diepenbeek, Belgium
}

(Received 26 March 2009; published 8 May 2009)

\begin{abstract}
We studied the transport properties of highly boron-doped nanocrystalline diamond thin films at temperatures down to $50 \mathrm{mK}$. The system undergoes a doping-induced metal-insulator transition with an interplay between intergranular conductance $g$ and intragranular conductance $g_{0}$, as expected for a granular system. The conduction mechanism in the case of the low-conductivity films close to the metal-insulator transition has a temperature dependence similar to Efros-Shklovskii type of hopping. On the metallic side of the transition, in the normal state, a logarithmic temperature dependence of the conductivity is observed, as expected for a metallic granular system. Metallic samples far away from the transition show similarities to heavily borondoped single-crystal diamond. Close to the transition, the behavior is richer. Global phase coherence leads in both cases to superconductivity (also checked by ac susceptibility), but a peak in the low-temperature magnetoresistance measurements occurs for samples close to the transition. Corrections to the conductance according to superconducting fluctuations account for this negative magnetoresistance.
\end{abstract}

DOI: 10.1103/PhysRevB.79.201203

PACS number(s): 73.23.Hk, 71.30.+h, 73.22.-f, 73.61.Cw

Depending on the microscopic parameters, a granular material can vary from a relatively good metal to an insulator, by changing the strength of electron tunneling coupling between conducting grains. Only recently, new insights have been gained on the theoretical understanding of such granular systems, ${ }^{1}$ which have been applied to the experimental low-temperature transport behavior in highly boron-doped nanocrystalline diamond (B:ncd). Indeed, renewed interest in doping-induced electronic phase transitions came up as superconductivity was discovered in heavily boron-doped diamond ${ }^{2}$ and was confirmed by several other groups (see Ref. 3 and references therein).

The metal-insulator transition in highly boron-doped diamond has been analyzed in ion-implanted films ${ }^{4}$ and more recently in single-crystalline diamond. ${ }^{5}$ In this Rapid Communication, we report on the metal-insulator transition and superconductivity in highly B:ncd thin films, demonstrating the influence of the granular structure on the transport properties at low temperatures.

Nanocrystalline diamond films were grown in a microwave plasma-enhanced chemical vapor deposition (MPCVD) as described elsewhere. ${ }^{6}$ Doping with boron was achieved by adding trimethylboron to the gas phase with molar concentrations $B / C$ reaching $10000 \mathrm{ppm}$. These films were grown on quartz substrates (suitable for transport measurements), which were either prepared by mechanical polishing with diamond nanoparticles (Bmec prefix) or by ultrasonic seeding in an aqueous colloid of $10 \mathrm{~nm}$ sized diamond powder (Bus prefix). ${ }^{7}$ Electrical resistivity measurements were performed from room temperature down to $360 \mathrm{mK}$ using a Quantum Design physical properties measurements system. A four-terminal configuration has been used for the electric contact to the films. In the same setup, magnetoresistance measurements were performed with perpendicular fields up to $8 \mathrm{~T}$. A dilution fridge was used at lower temperatures with perpendicular fields up to $14 \mathrm{~T}$. The change in the mutual inductance of a small coil was used for the ac magnetic susceptibility measurements. The boron concentration $n_{B}$ has been obtained from secondary ion mass spectroscopy (SIMS) depth profiles of ${ }^{11} B^{-},{ }^{12} C^{-}$, and ${ }^{11} B^{12} C^{-}$ions.

The temperature dependence of the normalized resistance $\rho / \rho(300 \mathrm{~K})$ in the range $300 \mathrm{~K}$ down to $350 \mathrm{mK}$ is shown in Fig. 1(a) for several samples (note the logarithmic scale for both resistance and temperature). The highly doped samples with $n_{B} \geq 1.3 \times 10^{21} \mathrm{~cm}^{-3}$ also show superconductivity with critical temperatures up to $T_{c}=2.1 \mathrm{~K}$. ac susceptibility measurements confirmed that this superconductivity has no filamentary nature. As illustrated by Fig. 1(b), the onset of the diamagnetic screening corresponds to the transition to zero resistance. The critical boron concentration $n_{c}$ for the metalinsulator transition is about $2.6 \times 10^{20} \mathrm{~cm}^{-3}$. 6 Similar values have been found in the case of single crystal and polycrystalline diamond. ${ }^{3}$ However, as will be shown below, the metal-insulator transition is not only due to the increasing doping within the grains but also to the increase in intergranular coupling between metallic grains.

Figure 2(a) shows the temperature dependence of the normalized conductivity (logarithmic scale) for the weakly doped samples Bus100 and Bmec2500, which are close to the metal-insulator transition. An Efros-Shklovskii (ES) type of hopping dependence following $\ln (\sigma) \propto\left(T_{0} / T\right)^{0.5}$ is observed over a remarkably wide range of temperatures, from 300 down to $20 \mathrm{~K}$. Multiple cotunneling processes can lead to Efros-Shklovskii-type variable range hopping in granular metal arrays with weak intergrain coupling. ${ }^{8,9}$ An estimate of the localization length $\xi$ from the experimentally determined $T_{0}$ values gives $\xi \approx 1 \mathrm{~nm}$. Taking into account an acceptable variation in the dielectric function for this estimate, the localization length would be still much smaller than the grain 

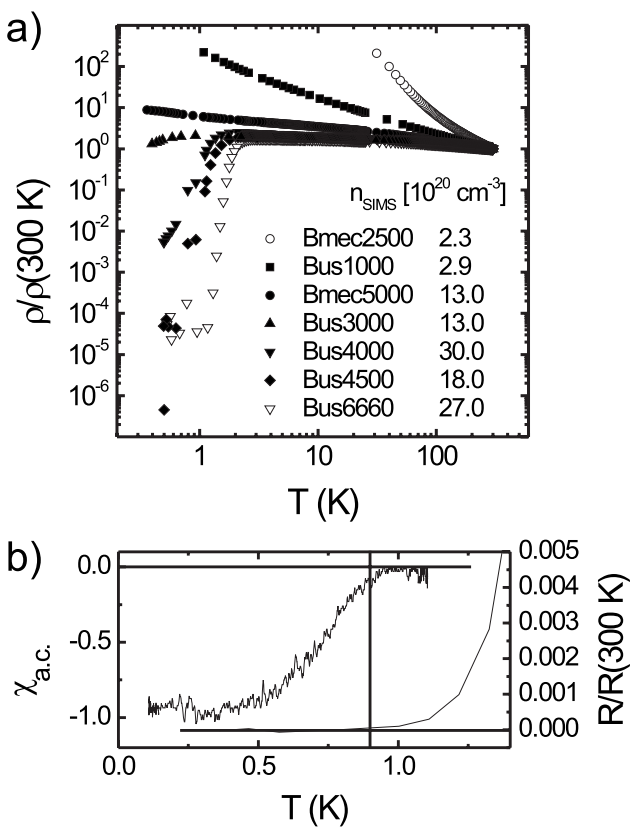

FIG. 1. (a) Temperature dependence of the normalized resistance $\rho / \rho(300 \mathrm{~K})$ from 300 down to $350 \mathrm{mK}$ (both in logarithmic scale). The weakly doped insulating sample Bmec2500 shows thermally activated transport. The higher doped metallic samples show also superconductivity; (b) ac magnetic susceptibility measurements clearly indicate the nonfilamentary nature of superconductivity. The onset of the diamagnetic response corresponds to the transition to zero resistance.

size in our samples. The situation corresponds to wellcoupled insulating grains (in agreement with the SIMS values) rather than weakly coupled metallic grains, with the localization length comparable to the interatomic distance between the impurities. ${ }^{10}$ However, Efros-Shklovskii type of temperature dependence at such high temperatures has not been observed in single-crystal diamond samples within the same doping range, where Mott variable range hopping has been seen without a crossover to ES hopping at low temperatures. ${ }^{5}$ This remains an open question which one can probably assign to the granularity of the samples.

The physical quantities characterizing the transport in a granular material are the dimensionless intragranular conductance $g_{0}$ and the intergranular (tunneling) conductance $g$. Both correspond to one spin component and are measured in the units of the quantum conductance $e^{2} / \hbar: g=G /\left(2 e^{2} / \hbar\right)$ with $G$ as the conductance $\left([G]=\Omega^{-1}\right)$. Other important parameters are the grain charging energy $E_{c}=e^{2} / 4 \pi k_{B} \epsilon_{0} \epsilon_{r} d(d$ is the grain size) and the mean level spacing $\delta=(\nu V)^{-1}$ ( $\nu$ is the density of states and $V$ is the volume of the grain). ${ }^{1}$ If the constituent grains are made of a superconductor material, the behavior of such a system can be quantified by adding one more energy parameter, the superconducting gap $\Delta$ of the material of a single grain. The interplay between Josephson coupling $J$ and grain charging energy $E_{c}$ determines whether global macroscopic superconductivity is built up in the sample or not. ${ }^{1}$ A "granular system" is obtained in the limit $g \ll g_{0}$, whereas the case $g \sim g_{0}$ can be viewed as a homogeneously disordered system.
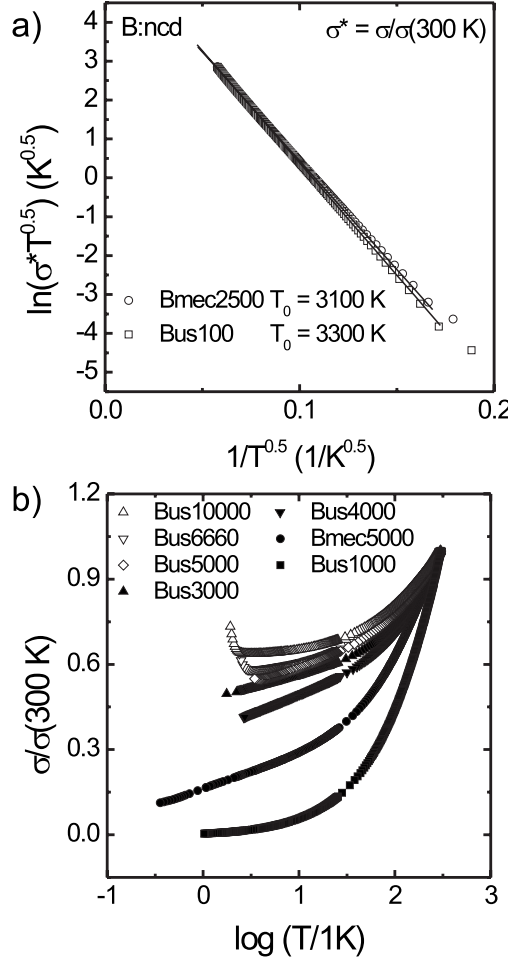

FIG. 2. (a) Temperature dependence of the normalized conductivity (logarithmic scale) for the weakly doped samples Bus100 and Bmec2500, following a Efros-Shklovskii hopping over a wide range of temperatures. The $T_{0}$ values of both samples are indicated; (b) logarithmic temperature dependence of the normalized conductivity for several highly doped samples as expected for a granular metal ( $T$ is in units of kelvin).

Samples with $g>g_{c}$ exhibit metallic transport whereas samples with $g<g_{c}$ show insulating behavior, corresponding to metallic grains with strong and weak intergrain couplings, respectively. Here, $g_{c}$ is the critical conductance which is related to $E_{c}$ and $\delta$ as

$$
g_{c}=(1 / 2 \pi D) \ln \left(E_{c} / \delta\right),
$$

where $D$ is the dimensionality. ${ }^{1}$ For a grain size $d \approx 150 \mathrm{~nm}$ and a typical density of states $\nu \approx 10^{20} \mathrm{eV}^{-1} \mathrm{~cm}^{-3}$ one gets $E_{c} \approx 20 \mathrm{~K}$ and $\delta \approx 1 \mathrm{mK}$. One could expect two-dimensional effects, as the grain size is on the same order of magnitude as the thickness and, thus, $g_{c} \approx 0.52$ or $g_{c} \approx 0.77$ for a three-dimensional or twodimensional system, respectively. These values lie between the estimated conductances for samples Bus100 and Bus1000.

For samples with $g>g_{c}$, one should find a logarithmic temperature dependence (for two as well as threedimensional systems) of the conductivity at low temperatures. ${ }^{1}$ This behavior has been reported for several granular metallic systems. ${ }^{11,12}$ As can be seen in Fig. 2(b), some of the nanocrystalline samples show a logarithmic temperature dependence at low temperatures, clearly indicating a metallic behavior in the granular limit $g \ll g_{0}$. A crossover to superconducting behavior occurs for the highly conducting samples (Bus4000 to Bus10000). The temperature at which 


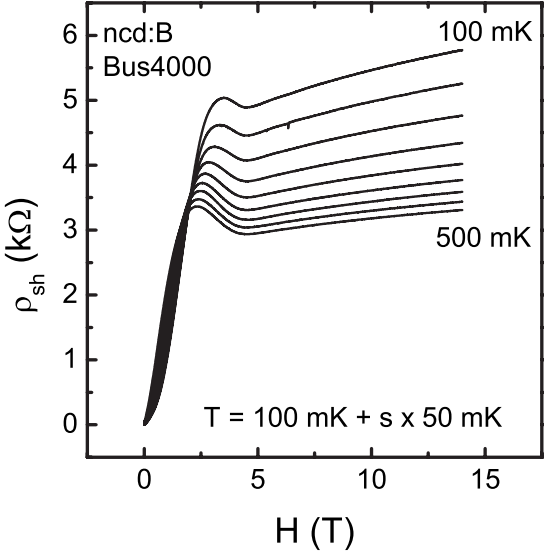

FIG. 3. Magnetoresistance of sample Bus 4000 at several constant temperatures, revealing regions with negative and positive magnetoresistance, respectively.

the deviation from the logarithmic temperature dependence takes place increases with doping. Electron-electron interaction is responsible for the logarithmic temperature dependence; at higher temperatures interactions with phonons lead to a power-law dependence (whole temperature range for Bus1000).

The superconducting properties of a granular system are in many ways determined by the properties of the grains. The superconducting gap $\Delta$ exists in each grain and its value is close to the gap magnitude in the bulk, provided $\Delta \gg \delta .{ }^{1}$ Anderson ${ }^{13}$ showed that the superconducting gap is suppressed or can even be fully destroyed if the latter inequality is not satisfied. In our case, $\delta=(\nu V)^{-1} \approx 1 \mathrm{mK}$ and $\Delta$ $\approx 300 \mu \mathrm{V}=3.6 \mathrm{~K}$ (Ref. 14) and, thus, these effects can be neglected. Sufficiently strongly coupled grains $(g \gg 1)$ can maintain (global) superconducting coherence because the coupling reduces the phase fluctuations.

In the approach of Chakravarty et al. (see Ref. 15) the originally strong Coulomb interaction is reduced by the electron tunneling to other grains. This renormalization corresponds to the screening of the Coulomb interaction by free charges usually seen in metals. In the case of strong coupling $(g \gg 1)$ and strong Coulomb interaction $\left(E_{c} \gg \Delta\right)$ the charging energy is renormalized to $\approx \Delta / g$. Therefore, for $g \gg 1$ the effective Coulomb energy is always smaller than the Josephson coupling $J \approx g \Delta$, and superconductivity occurs at sufficiently low temperatures. ${ }^{16,17}$ With $E_{c} \approx 20 \mathrm{~K}$ this is the case for the most highly doped samples. These samples follow a transport behavior typically seen for disordered metals. Measurements of the magnetoresistance as well as the critical magnetic field phase diagram reveal similar results compared to highly boron-doped single-crystal diamond. ${ }^{5}$

Figure 3 shows the magnetoresistance of sample Bus 4000 at several constant low temperatures in the range from 100 up to $500 \mathrm{mK}$ and with perpendicular fields up to $14 \mathrm{~T}$. Bulk superconductivity (superconducting grains) is destroyed by the application of extremely strong fields; the positive magnetoresistance in the high-field limit resembles the behavior seen in metallic single-crystal and nanocrystalline diamond films. ${ }^{18,19}$ By decreasing the magnetic field a region with negative magnetoresistance is found. Only at sufficiently weak magnetic fields the resistivity starts to decrease and the sample displayed superconducting behavior. Similar behaviors have been reported in other granular systems. ${ }^{20-22}$

One possibility to explain the negative magnetoresistance of a granular metal is due the suppression of the density of states (DOS) due to superconducting fluctuations. ${ }^{23}$ One fluctuation contribution comes from nonequilibrium Cooper pairs forming a new transport channel $\left(\delta \sigma_{\mathrm{AL}}\right),{ }^{24}$ and another from coherent scattering of electrons forming a Cooper pair on impurities $\left(\delta \sigma_{\mathrm{MT}}\right){ }^{25,26}$ Both corrections enhance conductivity and lead to a positive magnetoresistance. The formation of nonequilibrium Cooper pairs results in a fluctuational gap in the one-electron spectrum. ${ }^{27}$ This suppression of the density of states has been shown to lead to a correction of the conductivity $\delta \sigma_{\text {DOS. }}{ }^{23}$ While in conventional superconductors $\delta \sigma_{\mathrm{AL}}$ and $\delta \sigma_{\mathrm{MT}}$ are more important than $\delta \sigma_{\mathrm{DOS}}$, in granular systems the DOS correction plays a very important role..$^{23,28}$ The conductivity $\delta \sigma_{\text {DOS }}$ is negative and its absolute value decreases with increasing magnetic field, thus leading to the negative magnetoresistance. This is what is seen for sample Bus4000 in Fig. 3.

Another possibility is based on the fact that, as is the case for sample Bus4000, $g$ is close to 1, approaching the quantum resistance $R_{0}=h /(2 e)^{2} \approx 6.4 \mathrm{k} \Omega$ for which a superconductor-insulator transition is expected. In the case of a superconductor-insulator transition, similar behaviors of the magnetoresistance have been reported. ${ }^{29-31}$ The origin of such a transition is still under debate. Some theories claim that it may be understood in terms of Cooper-pair scattering out of the superconducting condensate into a Bose glass, ${ }^{32,33}$ while others claim that a percolation description of the superconductor-insulator transition is more adequate. ${ }^{34}$

In summary, we report on the metal-insulator transition in highly boron-doped nanocrystalline diamond films. The metal-insulator transition is induced by the increasing doping, thereby also controlling the intragranular conductance. Low-temperature transport measurements suggest that metallic nanocrystalline diamond follows dependencies for the conductivity typical for a granular system with metallic/ superconducting grains and strong or low intergranular coupling. On the insulating side of the transition, EfrosShklovskii-type variable range hopping was observed, while on the metallic side a logarithmic temperature dependence of the conductivity was found. The granular structure influences the superconducting properties and the magnetoresistance in highly boron-doped metallic nanocrystalline diamond films. Tuning of the microscopic parameters leads to rich behavior and similarities to the superconductor-insulator transition are pointed out, explained on the basis of superconducting fluctuations and the importance of granularity.

This work was partly financially supported by the Research Programs G.0068.07 and G.0430.07 of the Research Foundation Flanders (FWO), the IAP-P6/42 project "Quantum Effects in Clusters and Nanowires," and by Nanosystems Initiative Munich, NIM. 
*philipp.achatz@grenoble.cnrs.fr

${ }^{1}$ I. S. Beloborodov, A. V. Lopatin, V. M. Vinokur, and K. B. Efetov, Rev. Mod. Phys. 79, 469 (2007).

${ }^{2}$ E. A. Ekimov, V. A. Sidorov, E. D. Bauer, N. N. Mel'nik, N. J. Curro, J. D. Thompson, and S. M. Stishov, Nature (London) 428, 542 (2004).

${ }^{3}$ E. Bustarret, P. Achatz, B. Sacépé, C. Chapelier, C. Marcenat, L. Ortéga, and T. Klein, Philos. Trans. R. Soc. London, Ser. A 366, 267 (2008)

${ }^{4}$ T. Tshepe, C. Kasl, J. F. Prins, and M. J. R. Hoch, Phys. Rev. B 70, 245107 (2004).

${ }^{5}$ T. Klein, P. Achatz, J. Kacmarcik, C. Marcenat, F. Gustafsson, J. Marcus, E. Bustarret, J. Pernot, F. Omnes, Bo E. Sernelius, C. Persson, A. Ferreira da Silva, and C. Cytermann, Phys. Rev. B 75, 165313 (2007)

${ }^{6}$ W. Gajewski, P. Achatz, O. A. Williams, K. Haenen, E. Bustarret, M. Stutzmann, and J. A. Garrido, Phys. Rev. B 79, 045206 (2009).

${ }^{7}$ O. A. Williams, O. Douhéret, M. Daenen, K. Haenen, E. Osawa, and M. Takahashi, Chem. Phys. Lett. 445, 255 (2007).

${ }^{8}$ T. B. Tran, I. S. Beloborodov, X. M. Lin, T. P. Bigioni, V. M. Vinokur, and H. M. Jaeger, Phys. Rev. Lett. 95, 076806 (2005).

${ }^{9}$ M. V. Feigel'man and A. S. Ioselevich, JETP Lett. 81, 227 (2005).

${ }^{10}$ B. I. Shklovskii and A. L. Efros, Electronic Properties of Doped Semiconductors (Springer-Verlag, Berlin, 1988).

${ }^{11}$ C. Radhakrishnan, C. K. Subramaniam, S. Sankaranaranyanan, G. V. S. Rao, and R. Srinivasen, Physica C 167, 53 (1990).

${ }^{12}$ L. Rotkina, S. Oh, J. N. Eckstein, and S. V. Rotkin, Phys. Rev. B 72, 233407 (2005).

${ }^{13}$ P. W. Anderson, J. Phys. Chem. Solids 11, 26 (1959).

${ }^{14}$ B. Sacépé, C. Chapelier, C. Marcenat, J. Kacmarcik, T. Klein, M. Bernard, and E. Bustarret, Phys. Rev. Lett. 96, 097006 (2006).

${ }^{15}$ S. Chakravarty, S. Kivelson, G. T. Zimanyi, and B. I. Halperin,
Phys. Rev. B 35, 7256 (1987).

${ }^{16}$ A. I. Larkin and Y. N. Ovchinnikov, Phys. Rev. B 28, 6281 (1983).

${ }^{17}$ U. Eckern, G. Schön, and V. Ambegaokar, Phys. Rev. B 30, 6419 (1984).

${ }^{18}$ E. Bustarret, E. Gheeraert, and K. Watanabe, Phys. Status Solidi A 199, 9 (2003).

${ }^{19}$ M. Nesládek, D. Tromson, C. Mer, P. Bergonzo, P. Hubik, and J. J. Mares, Appl. Phys. Lett. 88, 232111 (2006).

${ }^{20}$ A. Gerber, A. Milner, G. Deutscher, M. Karpovsky, and A. Gladkikh, Phys. Rev. Lett. 78, 4277 (1997).

${ }^{21}$ V. F. Gantmakher, M. Golubkov, J. G. S. Lok, and A. Geim, JETP Lett. 82, 951 (1996).

${ }^{22}$ R. Parthasarathy, X.-M. Lin, K. Elteto, T. F. Rosenbaum, and H. M. Jaeger, Phys. Rev. Lett. 92, 076801 (2004).

${ }^{23}$ I. S. Beloborodov and K. B. Efetov, Phys. Rev. Lett. 82, 3332 (1999).

${ }^{24}$ L. G. Aslamazov and A. I. Larkin, Sov. Phys. Solid State 10, 875 (1968)

${ }^{25}$ K. Maki, Prog. Theor. Phys. 39, 897 (1968).

${ }^{26}$ R. S. Thompson, Phys. Rev. B 1, 327 (1970).

${ }^{27}$ E. Abrahams, M. Redi, and J. Woo, Phys. Rev. B 1, 208 (1970).

${ }^{28}$ I. S. Beloborodov, K. B. Efetov, and A. I. Larkin, Phys. Rev. B 61, 9145 (2000).

${ }^{29}$ A. F. Hebard and M. A. Paalanen, Phys. Rev. Lett. 65, 927 (1990).

${ }^{30}$ M. A. Paalanen, A. F. Hebard, and R. R. Ruel, Phys. Rev. Lett. 69, 1604 (1992).

${ }^{31}$ N. Mason and A. Kapitulnik, Phys. Rev. Lett. 82, 5341 (1999).

${ }^{32}$ M. P. A. Fisher, Phys. Rev. Lett. 65, 923 (1990).

${ }^{33}$ E. S. Sorensen, M. Wallin, S. M. Girvin, and A. P. Young, Phys. Rev. Lett. 69, 828 (1992).

${ }^{34}$ Y. Dubi, Y. Meir, and Y. Avishai, Phys. Rev. B 71, 125311 (2005). 